\title{
Dementia Assessment and Management Protocol for Doctors in Nepal
}

\author{
Arun Jha, ${ }^{1}$ Nidesh Sapkota ${ }^{2}$ \\ 'Mental Health services for Older People, Hertfordshire Partnership NHS Foundation Trust, UK, ${ }^{2}$ Department of Psychiatry, \\ BPK Institute of Health Sciences, Dharan, Nepal.
}

\begin{abstract}
WHO Dementia Report published in 2012 highlighted the exponential rise of dementia in the elderly population worldwide, especially in low-and middle-income countries. There may be over 135,000 people with dementia in Nepal, but there are no formal arrangements for diagnosis and treatment or any care and support for their caregivers.
\end{abstract}

WHO developed the Mental Health Gap Action Programme (mhGAP) Intervention Guide in 2010, for mental, neurological and substance use, including dementia in non-specialist health settings. This paper reviews the current dementia care provisions in Nepal and proposes a mhGAP-based dementia assessment and management protocol for Nepalese doctors in hospital/clinic settings. The protocol also outlines a post-diagnostic advice for dementia caregivers. The protocol would be discussed at various stakeholders meetings before recommending for routine clinical use.

Keywords: caregiver burden; dementia; MCI; memory clinic; mhGAP-IG; protocol.

\section{INTRODUCTION}

Dementia (renamed 'Major Neurocognitive Disorder' in DSM-5) is a chronic and progressive neurodegenerative syndrome characterised by global deterioration in intellectual and social functioning that requires, at different points, medical, social or mental health interventions. The prevalence of Alzheimer's disease and other dementias will continue to increase with the rapid growth of older population worldwide. Diagnosing and managing these complex conditions is a challenge for health care professionals in resource-poor countries. The WHO report on dementia published in April 2012 highlights the serious consequences of dementia in low- and middle-income countries (LMICs), and offers important statistics and key messages. ${ }^{1}$ In 2010, dementia affected 35.6 million people worldwide -0.5 percent of the world's population. This number will double by 2030 and will more than triple by 2050 .
Currently, $58 \%$ of people with dementia live in LMICs, and this proportion is projected to increase to $71 \%$ by 2050. Around the world a new case of dementia occurs every four seconds, which is equivalent of 7.7 million new cases each year. Dementia is overwhelming for the caregivers and adequate support is required for them from the health, social, financial and legal systems. Although there is no cure for dementia, timely diagnosis and early intervention can help people to steer a course through the 7-12 years they may live with dementia, and will assist them to avoid crises and promote their wellbeing. ${ }^{1}$

According to the 2011 census, conducted by the Central Bureau of Statistics of Nepal, the population of people aged 60 years and above, also called senior citizens,

Correspondence: Dr. Arun Jha, Consultant Old Age Psychiatrist, Logandene Care Unit, Ashley Close, Hemel Hempstead, Hertfordshire, HP3 8BL, UK. Email: arunjhauk@gmail.com. 
was 2.2 million, accounting for 8.3 percent of Nepal's total population of 26,494504 . The majority of senior citizens belong to age group of 60-74 years (Figure 1). These people are at a higher risk of developing dementia in next few years. Based on recent epidemiological studies in LMICs, prevalence rate of dementia can be extrapolated for Nepal. Assuming the prevalence rate of dementia as $3 \%$ among $60+$ Nepalese senior citizens, compared to $5 \%$ in 65 + population of $\mathrm{LMICs}^{2}$ at least 66,000 people should have some form of dementia. This figure will double in next 20 years. A recent status report on elderly people, ${ }^{3}$ acknowledges the fact that most Nepalese enter old age after a lifetime of poverty and deprivation, poor access to health care and a diet that is usually inadequate in quality and quantity. It is estimated that over $80 \%$ of elderly in Nepal live with their children, especially with their sons because of cultural and religious reasons. However, younger generations are moving away leaving their older parents lonely and vulnerable.

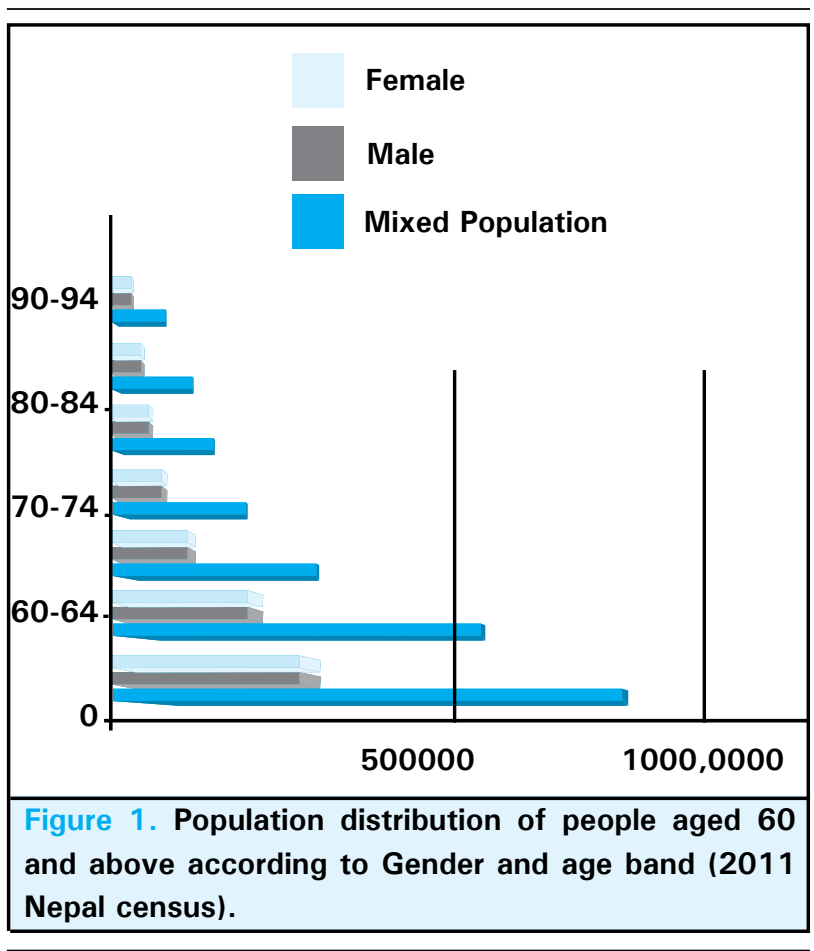

The impact of dementia upon the individual, the family and the society is immense in terms of disability, dependency, caregiver strain and costs. ${ }^{2}$ Many people with dementia need long-term care, currently provided by family caregivers. Primary care services do not meet their needs. Governments do not provide long-term care, nor support caregivers. Caregiver's psychological and economic strain is as high as in the developed world, despite traditional family care arrangements. This paper reviews the current state of dementia care in Nepal, discusses the relevance of WHO Dementia Report in
Nepalese context, and proposes a simple protocol for diagnosis and managing dementia for doctors.

\section{LESSONS FROM RECENT RESEARCH}

There has been no epidemiological survey of dementia in Nepal. A preliminary survey done in Tikathali, Lalitpur revealed that almost $50 \%$ of people over aged of 60 were suffering from memory related problems. ${ }^{4}$ The prevalence rate of this survey is too high to be accepted; and no meaningful conclusions can be drawn since the methodology of the survey is not available.

Traditionally, diagnosis of dementia is made using international criteria such as DSM IV or ICD-10, but DSMIV dementia is particularly rare in Indian subcontinent. The prevalence of DSM-IV dementia varies from less than one percent in the least developed countries (India and rural Peru) to $6.4 \%$ in Cuba. ${ }^{5}$ On the other hand, studies using 10/66-research group's criteria have fond dementia prevalence to be double that of DSMIV dementia, and varied between $5.6 \%$ and $11.7 \%$. The discrepancy was explained by the observation that informants in the least developed sites, particularly India, were less likely to report cognitive decline and social impairment (an essential criterion for DSM-IV dementia diagnosis) even in the presence of objective memory impairment. Secondly, objective cognitive impairment may be less likely to lead to noticeable impairment in the performance of normal social roles because of the high level of instrumental support routinely provided to all older people, particularly in the early stages of dementia. The third reason offered by the $10 / 66$ researchers is that informants may have noted the impairment/decline, but they may have been reluctant to disclose this because of the culture of respect towards older people. Fourth reason is low awareness; impairment/decline may have been noted, but attributed to 'normal ageing' and hence not worthy of mention given the implicit focus of the assessment upon abnormality. Barriers to improvement of dementia diagnosis and care are similar to those confronting mental health services. Older people in Nepal often present with memory problems due to depression and other treatable conditions that remain undiagnosed and untreated. ${ }^{6}$

\section{DEMENTIA CARE IN NEPAL}

Nepal is not at all prepared for providing dementia care needs of its people. In the early stages of the disease, mental health resources play an important role in the detection of cognitive impairment and diagnosis of dementia. That is why WHO has grouped dementia together with mental, neurological and substance abuse disorders, but in LMICs there are not enough mental 
health resources or professionals. In Nepal, there are only 0.13 psychiatrists, 0.27 nurses, and 0.19 psychosocial providers per 100,000 population, and the suggested numbers required are 288, 2928, and 2549 respectively. ${ }^{7}$ In the current political situation, Nepal is unlikely to meet these expectations in the near future.

Developing effective approaches for diagnosis and post-diagnostic support are the initial steps towards providing adequate services for people with dementia and their family. ${ }^{8}$ The 10/66 Dementia Research Group's culture- and education-fair diagnostic protocol is validated in many $\mathrm{LMICs}^{9}$ but their diagnostic algorithm is too lengthy and complicated to be used routinely. Moreover, better and royalty-free cognitive assessment instruments have been introduced in last few years. More importantly, WHO developed the Mental Health Gap Action Programme (mhGAP) to scale up care for mental, neurological (including dementia) and substance use in LMICs. ${ }^{10}$ The mhGAP intervention guide (IG), ${ }^{11}$ contains simple clinical algorithms for doctors and nurses in non-specialist health settings.

The 10/66 Group has been testing the effectiveness of training community healthcare workers to identify people with dementia and deliver a brief intervention to educate and train caregivers. ${ }^{12}$ They recommend such interventions to be incorporated into horizontally constructed programs addressing the generic needs of frail, dependent older people and their caregivers, whether arising from cognitive, mental or physical disorders. ${ }^{2}$ The Government of Nepal is committed to promote dementia and mental health care through the national NCD strategy that requires implementation. To address these glaring gaps in the services in the short term, we propose a protocol for diagnosing and managing dementia as well as educating and supporting families.

\section{DIAGNOSTIC AND POST-DIAGNOSTIC DEMENTIA CARE PROTOCOL}

Based on the mhGAP's Dementia assessment and management guide (p 50-56), ${ }^{11}$ we have developed a simple clinic-based diagnostic procedure for doctors (primarily psychiatrists and neurologists) in conjunction with a training and education guide for caregivers. We have adapted the mhGAP dementia-IG and the Montreal Cognitive Assessment (MoCA) instrument, ${ }^{13}$ to suit Nepalese context. MoCA takes only 10 minutes to complete, includes tests for executive functions, and is free for nonprofit use. We have also included a guide to diagnose Mild Cognitive Impairment $(\mathrm{MCl}$, to be called 'Minor Neurocognitive Disorder' in DSM-5), as people with amnestic form of $\mathrm{MCl}$ are at a higher risk of developing Alzheimer's disease. A recent study in LMICs has found that the prevalence of $\mathrm{MCl}$ is relatively high $(4.3 \%)$ in Indian subcontinent. ${ }^{14}$

\section{DIAGNOSTIC PROCEDURE}

Identifying dementia requires assessment of memory and cognitive functioning using simple tests, and confirming by interviewing a reliable informant to establish the onset and course of the illness, and difficulty in carrying out daily work and domestic or social activities. An outline of a 'Dementia Assessment and Management Protocol' for Nepalese psychiatrists (Table 1).

Table 1. Dementia assessment and management protocol for Nepal adapted from mhGAP-IG. ${ }^{11}$

A. Presenting problems - Ask the person or family about the symptoms:

When were they first noticed?

What was the age of the person?

Was the onset sudden or gradual (over several months and years)?

What is the duration of the illness?

Is their associated drowsiness, impairment of consciousness?

Does he/she get confused at night, wander about, talk nonsense?

Was the onset linked to a head injury, a blackout or a stroke?

Medical History - Is there a clinical history of:

Alcohol dependence?

Goiter, slow pulse, dry skin or hypothyroidism?

Sexually transmitted infection or HIV?

Cardiovascular risk factors? (hypertension, hyperlipidaemia, diabetes, smoking, obesity, heart disease, previous stroke or transient ischaemic attacks) poor dietary intake, malnutrition, anaemia?

Cognitive Test - Subjective Memory Impairment Ask the person:

1. Have you had any difficulty with your memory $(0$, no; 1 , yes $)$ ?

2. Have you tended to forget names of your family or close friends $(0$, no/transient; 1 , noticed most days per week; 2, noticed daily)?

3. Have you tended to forget where you have put things $(0$, no/transient; 1 , noticed most days per week; 2, noticed daily)?

4. Do you have to make more efforts to remember things than you used to $(0$, no; 1 , yes $)$

Note: Subjective memory impairment can be defined as present when an individual scores 3 or more. 
A. Cognitive Test - Objective Memory Impairment or any other locally validated tool should be considered if the tests in this section indicate cognitive impairment):

* Assess memory by asking the person to repeat three common words (e.g. velvet, temple, rose;) immediately, then again 3-5 minutes later

* Assess orientation to time (time of day, day of week, season and year), and place (where the person being tested, or where is the nearest market or store to their home)

B. Test language skills by asking the person to name parts of the body, and explaining the function of an item, such as, "what do you do with a hammer?"

C. Activities of living

Ask the family/relative a brief question about the patient's activities these days (Score 0 for 'No' and 1 for 'yes':

* Has there been a general decline in his/her mental functioning?

* Have you noticed a change in her ability to think and reason?

* Does she often forget where she has put thinghs?

* Does she sometimes forget what happened the day before?

* Does she sometimes forget where she is?

* Does she have difficulty dressing, feeding or toileting? (If because of physical disability then code 0)

TOTAL SCORE (MAXIMUM $<=6$ ):

B. Mental and physical state

* (Depression) Depressed mood, early morning wakening, poor appetite and suicidal thoughts

* (Psychosis): hallucinations and delusions

* (Physical health): pain, constipation, urinary infection, side-effects of medication

E. Carer's strain

Assess whether the family caregivers:

* Find it particularly difficult to manage?

* Are coping, experiencing strain, or depressed?

* Are facing loss of income and/or additional expenses because of the needs for care?

B. Diagnosis and communication

Diagnosis of $\mathrm{aMCl}$ can be made on the basis of following criteria:

* Objective memory impairment beyond that expected for age (MoCA score $=22-25$ )

* Subjective memory complaint (SMI score >3)

* No, or only mild impairment in core activities of daily living

* No dementia.

In dementia there is chronic decline in memory and in at least one other cognitive function (language, visual-spatial, executive) sufficient to affect daily life. Diagnosis of dementia can be made if memory complaint or cognitive impairment:

* Has been present for at least 6 months

* Is progressive in nature, and

* Is associated with impairment in activities of daily living

Delirium is likely if the onset is abrupt and duration is short (days or week), if the disturbance is more at night and is associated with impairment of consciousness or awareness, and if there is disorientation to time/place. In MoCA the clock-drawing test is also abnormal.

In the absence of risk factors such as hypertension, diabetes, obesity, cerebrovascular event, and a slow and gradual course, a diagnosis of Alzheimer's disease (AD) can be made; patients with mild to moderate $A D$ should receive a trial of a cognitive enhancer, if pulse rate is normal and there is no history of gastric or duodenal ulcer. Patients should be referred to specialists if onset is before the age 60 , clinical hypothyroidism, cardiovascular disease, history of STD or HIV, or history of head injury or stroke is present. Communication: Explain the diagnosis of $\mathrm{MCl}$ or dementia to patients and family tailored to their ability to understand and retain information. Consider saying: People with $\mathrm{MCl}$ are at a higher risk of developing dementia later, but many don't

* Dementia is an illness of the brain and tends to get worse over time

* Although there is no cure, there is much that can be done to help and support the person and the family

* There are a few drugs available in the market to slow down the progress of Alzheimer's disease

* Many specific concerns and behaviours can be managed as they arise. A lot can be done to make the person more comfortable and to make life less stressful for the caregiver

Post-diagnostic training and support

The 10/66-caregiver education and training intervention 
is delivered over five, weekly, half hour sessions. ${ }^{12}$ In Nepal, currently, it is not possible to offer any community level education and training to dementia caregivers at a national level. Instead, those diagnosed with dementia at hospitals, may be offered information and advice individually or in groups. A model of postdiagnostic education and training protocol for people with dementia and family caregivers, based on mhGAP Intervention Guide (Table 2). ${ }^{11}$

Table 2. Post-diagnostic education and training protocol for dementia caregivers.

Following the diagnostic session, people with dementia and their families can be offered the following session either individually or in groups of other patients seen on the day. They may be offered a copy of the 'Dementia First Aid' book written in Nepali or directed to www.ardsnepal.org for free download.

A. Psychosocial interventions for cognitive symptoms and functioning

* Provide regular orientation to people with dementia to help them to remain orientated to time, place and person

* Use materials such as newspapers, radio or TV programmes, family albums and household items to promote communication, to orient them to current events, to stimulate memories and to enable people to share and value their experience

* Use simple short sentences to make verbal communication clear. Listen carefully to what the person has to say

* Keep things simple, avoid changes to routine and avoid exposing the person to unfamiliar and bewildering places unless this is necessary

B. Promoting independence, functioning and mobility Advice caregivers to:

* Use scheduled toileting and prompted toileting for incontinence

* Prompt regularly for food and fluid intake

* Avoid adversarial debates; try to redirect conversation instead

* Maintain a calm demeanour

* Offer graded assistance (as little help as possible to perform ADLs), role modelling, cueing and positive reinforcement to increase independence

* Make adaptations in the person's home such as hand-rails or ramps, signs of key locations (e.g. toilet, bath, bedroom) to ensure that the person does not get lost
* Encourage physical activity and exercise to maintain mobility and to reduce the risk of falls

* Consider recreational activities (tailored to the stage of dementia)

* Manage sensory deficits (such as low vision, poor hearing)

C. Managing behavioural and psychological symptoms Advice caregivers to:

* Identify and treat underlying physical health problems that may affect behaviour (pain, constipation, etc.)

* Simulate family presence with video or audio tapes

* Consider soothing, calming or distracting strategies, such as walking, listening to music, engaging in conversation etc.

* Pharmacological interventions - for extreme behavioural and psychological symptoms of dementia, consider antipsychotic medication such as low dose haloperidol (0.5 mg orally or i.m.)

\section{Intervention for carers}

* Acknowledge that it can be extremely frustrating and stressful to care for people with dementia.

* Provide training and support in specific skills (like managing difficult behaviour), if necessary

* Encourage seeking practical support and respite care from friends and relatives, so that the main caregiver can rest or carry out other activities

* Explore whether the person qualifies for any government or non-government benefits (e.g. ARDS Nepal Helpline)

* If depressed, carers may need counselling or treatment

\section{E. Follow-up care}

* Perform regular medical and social care review every three to six months

* Compare current assessment with previous notes, and discuss findings with the person and their carer

* Discuss and agree on any changes in the treatment plan

\section{DISCUSSION}

Do we need dementia diagnostic service in Nepal? It may be argued that a diagnosis of dementia would not be helpful in the absence of accessible evidence-based programmes of continuing care and support for people diagnosed and their families and caregivers. However, those seeking help at secondary care and teaching hospitals at least deserve diagnostic service and basic education and information about dementia. Dementia 
clinic in general hospital has been found successful in Kerala, India, and it is feasible to start weekly dementia clinics using existing infrastructure in developing countries. ${ }^{15}$ However, the Kerala model requires a multidisciplinary team, and uses a battery of tests, which may not be feasible elsewhere. The proposed Nepalese protocol has been adapted to suit the local situation and limited trained workforce. An evaluation study is being set up to investigate its acceptability and suitability in Nepalese health system, and its impact on caregiver's burden and quality of life. This protocol will be discussed at the annual scientific conference of the Psychiatric Association of Nepal (PAN) in April 2013. The Alzheimer's disease and related Dementias Society (www.ardsnepal.org) Nepal and PAN may join hands in capacity building for dementia care and in organising initial training and continued support and supervision to psychiatrists and other physicians interested in dementia care. Hopefully, the Ministry of Health and Population will take note of these initiatives and incorporate them into long term NCD plans and strategies. Dementia care in Nepal is unlikely to be available nationwide in the foreseeable future. We recommend that every teaching and zonal hospital should set up a memory clinic to serve local people. This may encourage people in rural areas to access services. A recent study, ${ }^{16}$ revealed that people in Nepal do not seek help because they do not feel sick enough to visit health facility; less than one third of sick people in rural areas access care at peripheral health facilities because of insufficient drug supply, staff unavailability, long distance to reach health institutions and inappropriate health facility hours. To raise public awareness of dementia, a grass root movement is needed in Nepal based on the model of Mental Health First Aid programme. ${ }^{17}$ A Dementia First Aid book has already been written in Nepali, and it is available for free download on www.ardsnepal.org, where other resources may be accessed.

\section{WAY FORWARD}

WHO dementia report has revealed the alarming magnitude of dementia-related problems in resourcepoor countries. In Nepal, number of people with dementia is likely to rise over 132,000 in next two decades. Nepal needs to prepare itself for this looming epidemic. This paper proposes a simple evidence based protocol for diagnosing and managing dementia in Nepal.

\section{ACKNOWLEDGEMENTS}

We would like to thank Professor K S Shaji, of Department of Psychiatry, Trissur Medical College, Kerala, India, for his valuable comments on the content of the manuscript.

\section{REFERENCES}

1. World Health Organization. Dementia: a public health priority. Geneva: WHO; 2012.

2. Prince $M$. The 10/66 dementia research group - 10 years on. Indian J Psychiatry 2009;51(Supple1):S8-15.

3. Geriatric Centre Nepal. Status report on elderly people $(60+)$ in Nepal on health, nutrition and social status focusing on research needs. [Online]. 2010 [cited 2013 Mar 10]. Available from: URL:http://www.globalaging.org/health/ world/2010/nepal.pdf

4. Shakya R. Dementia: forget me not. Ekantipur [newspaper online]. 2011 [cited 2013 Mar 10]. Available from: URL: http://www.ekantipur.com/2011/12/05/related-article/ dementia-forget-me-not/344991.html

5. Llibre Rodriguez JJ, Ferri CP, Acosta D, Guerra M, Huang Y, Jacob KS et al. Prevalence of dementia in Latin America, India, and China: a population-based cross-sectional survey. Lancet. 2008 Aug 9;372(9637):464-74.

6. Jha A, Adhikari SR. Mental health services in New Nepal observations, objections and outlook for the future. JNMA J Nepal Med Assoc. 2009 Apr-Jun;48(174):185-90.
7. Bruckner TA, Scheffler RM, Shen G, Yoon J, Chisholm D, Morris J et al. The mental health workforce gap in low- and middle-income countries: a needs-based approach. Bull World Health Organ. 2011 Mar 1;89(3):184-94.

8. Jha A, Jan F, Gale T, Newman C. Effectiveness of a recovery-orientated psychiatric intervention package on the wellbeing of people with early dementia: a preliminary randomised controlled trial. Int J Geriatr Psychiatry. 2012 Jul 30.

9. Prince M, Acosta D, Chiu H, Scazufca M, Varghese M. Dementia diagnosis in developing countries: a cross-cultural validation study. Lancet. 2003 Mar 15;361(9361):909-17.

10. World Health Organization. Mental Health Gap Action Programme: scaling up care for mental, neurological and sub- stance use disorders. Geneva: World Health Organization; 2008.

11. World Health Organization. mhGAP Intervention Guide for mental, neurological and substance misuse disorders in non-specialized health settings. Mental health Gap Action Programme. Geneva: WHO; 2010 .

12. Prince M, Ferri CP, Acosta D, Albanese E, Arizaga R, Dewey $\mathrm{M}$ et al. The protocols for the 10/66 dementia research group 
population-based research programme. BMC Public Health. 2007 Jul 20;7:165.

13. Nasreddine ZS, Phillips NA, Bédirian V, Charbonneau S, Whitehead V, Collin I et al. The Montreal Cognitive Assessment, MoCA: a brief screening tool for mild cognitive impairment. J Am Geriatr Soc. 2005 Apr;53(4):695-9.

14. Sosa AL, Albanese E, Stephan BC, Dewey M, Acosta D, Ferri $\mathrm{CP}$ et al. Prevalence, distribution, and impact of mild cognitive impairment in Latin America, China, and India: a 10/66 population-based study. PLoS Med. 2012 Feb;9(2):e1001170.
15. Shaji KS, Iype T, Praveenlal K. Dementia clinic in general hospital settings. Indian J Psychiatry. 2009 Jan;51(1):42-4.

16. Paudel R, Upadhyaya T, Pahari DP. People's perspective on access to health care services in a rural district of Nepal. JNMA J Nepal Med Assoc. 2012 Jan-Mar;52(185):20-4.

17. Jha A, Kitchener BA, Pradhan PK, Shyangwa P, Nakarmi B. Mental health first aid programme in Nepal. J Nepal Health Res Counc. 2012 Sep;10(22):258-60. 